\title{
A Method of Experts' Knowledge Approval for Industrial Safety Expertise Task
}

\author{
Alexander F. Berman \\ Matrosov Institute for System Dynamics and Control \\ Theory of Siberian Branch of the Russian Academy of \\ Sciences \\ Irkutsk, Russia \\ berman@icc.ru \\ Galina S. Maltugueva \\ Matrosov Institute for System Dynamics and Control \\ Theory of Siberian Branch of the Russian Academy of \\ Sciences \\ Irkutsk, Russia \\ gama@icc.ru
}

\begin{abstract}
The paper discusses a conception of a method of experts ' knowledge (opinions) approval for multidisciplinary tasks of industrial safety expertise of critical petrochemical objects. The proposed method is based on ontological models of a problem domain, as well as group decision-making methods. The main results of method are a model of a multidisciplinary task and its solution which are agreed by experts of various subject areas. The task model describes the concepts, laws of a subject area and solving methods. It is proposed to use the ARAMIS (Aggregation and Ranking Alternatives nearby the Multi-Attribute Ideal Situation) method to agreed experts' opinions. Also the paper presents examples of a model description for an identification task of technical states, and approval of experts' opinions at formalization of knowledge for a rule-based expert system intended for this task.
\end{abstract}

Keywords - ontology, problem ontology, task ontology, multidisciplinary approach, group decisionmaking methods, industrial safety expertise.

\section{INTRODUCTION}

The aging of technical devices (TD) in many industries is ahead of the rate of their modernization and replacement and proves the actuality of the problem of ensuring the safety of industrial facilities. This is especially true for long-term operated TD of critical infrastructures such as oil refineries, petrochemical and chemical enterprises. The safe operation of these enterprises requires the periodical assessment of their technical state (TS) of these TD by means of technical diagnostics, technical inspections in order to determine potentially dangerous elements and components, and making appropriate decisions to avoid catastrophic failures.

The quality of the TS assessment for industrial safety expertise (ISE) tasks can be improved by means of information and analytical support based on data analysis methods, artificial intelligence and specialized software. The main purpose of ISE is to confirm the compliance of the examinated object to the industrial safety requirements of the Russian

\author{
Olga A. Nikolaychuk \\ Matrosov Institute for System Dynamics and Control \\ Theory of Siberian Branch of the Russian Academy of \\ Sciences \\ Irkutsk, Russia \\ nikoly@icc.ru
}

Alexander Y. Yurin

Matrosov Institute for System Dynamics and Control Theory of Siberian Branch of the Russian Academy of Sciences

Irkutsk, Russia

iskander@icc.ru

Federation. The conclusion of ISE is entered to the Rostekhnadzor registry.

The tasks of ISE are multidisciplinary tasks, and its decision requires the use and processing of huge amounts of data and knowledge of various scientific disciplines. These disciplines are characterized by the following factors: heterogeneity of information and algorithms for its processing, different degrees of formalization and knowledge of subject areas, the level of training of users in the field of information technology, as well as in areas of related disciplines [1].

This problem has something in common with the tasks of reaching a consensus in the work of technical committees on standardization [2], group decision support systems (GDSS), collective intelligence [3], virtual heterogeneous teams [4], e-expertise [5,6]. The feature of this work is the focus on multidisciplinarity (as a result of the need to bring together specialists from different subject areas) and ability to use all available heterogeneous information for solving complex tasks in the case the collective discussions on the stages of setting particular tasks, choosing methods for solving them, etc. to solve a general problem.

An ontological model of a common multidisciplinary information space [7] and an algorithm for the self-organization of the decisionmaking process [8] are proposed for solving this problem in the form of decision support system for ISE tasks (Fig. 1):

$D S S=\left(E, D t, K n l, O n t, P, P^{h}, S l v, C r d, R_{D S S}, I n d, P l n, U I\right)(1)$

where $E$ are experts, $D t$ are databases, $K n l$ are knowledge bases, Ont is ontology of the subject and problem areas, Ont $\rightarrow\left\{P, P^{h}, D t, K n l, R_{D S S}\right\}, P$ are tasks, $P^{h}$ is a hierarchy of tasks, $S l v$ are "solvers", $R_{D S S}$ are relationships between $D S S$ components, in particular, between: experts and tasks, tasks, tasks and "coordinators", tasks and "solvers", $\mathrm{Crd}$ are "coordinators" of tasks, Ind are DSS status indicators, 

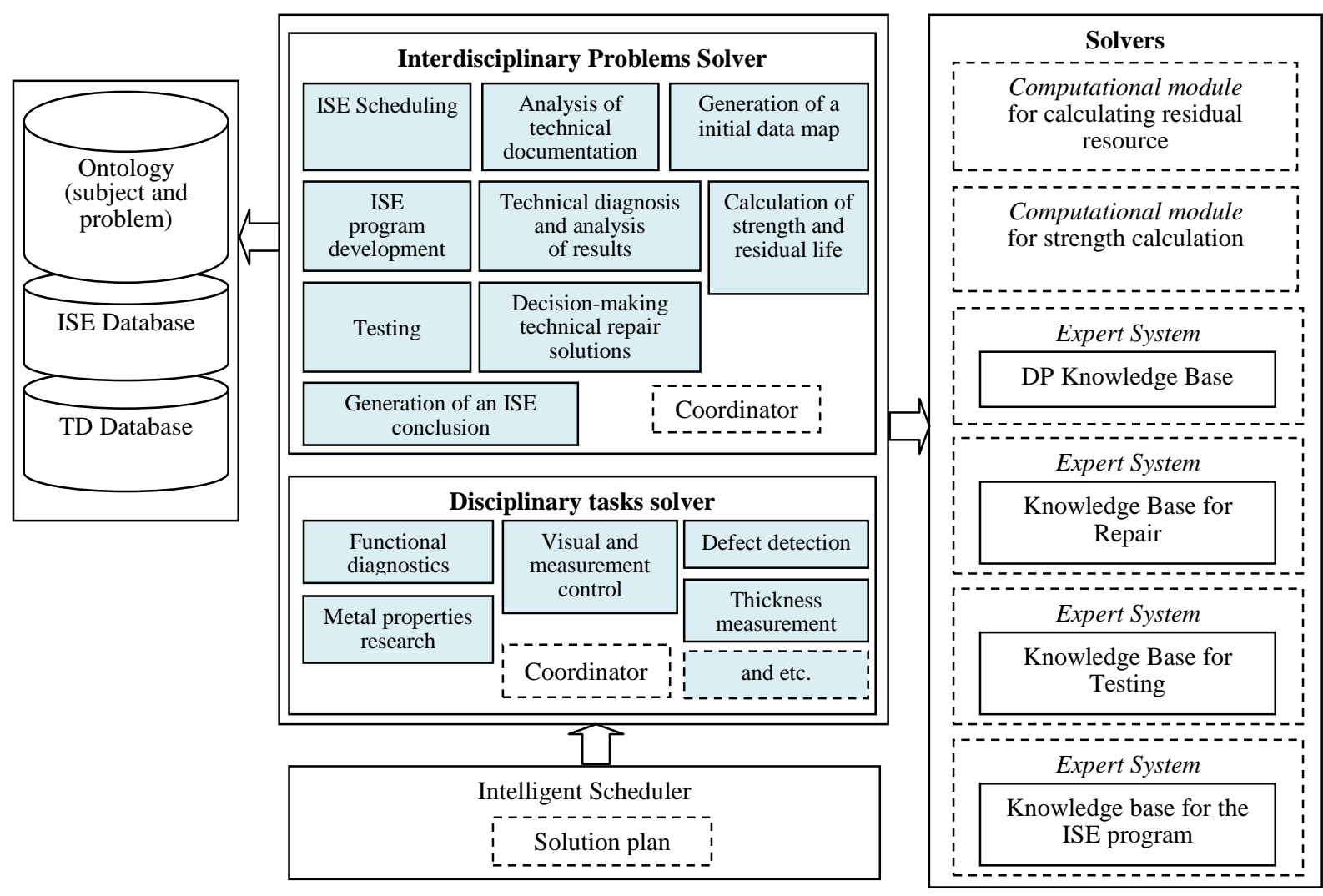

Fig. 1. Conceptual architecture of the decision support system for ISE

Ind $=\left\{I^{D e f P}, I^{S P}, I^{U n}\right\}$ is a set of indicators of: formulation, status, task incompleteness, $P \ln$ is a scheduler, that implements self-organizing algorithm SAlg for solving a multidisciplinary task on the basis of local rules LRule, $U I$ is a user interface.

The need to combine the efforts of experts from the most diverse subject areas (disciplines) for the formulation and solving multidisciplinary tasks determines the relevance of the task of coordinating their opinions (knowledge). This function is implemented in the system by "coordinators" of tasks Crd. The task of experts' knowledge agreement relates to a typical task of a decision-making theory. In this task a team selects one or more best objects (elements of the problem statement), which are characterized by heterogeneous signs. There are relatively few methods for group ordering of multi-character objects $[9,10]$. We single out the verbal decision-making methods (ARAMIS - method of Aggregation and Ranking Alternatives nearby the Multi-Attribute Ideal Situation, CLPRSS - method of CLosed PRocedures of Support Situations, etc.) that solve weakly structured tasks in which the preferences of several decision makers may be inconsistent and which allow one: to receive information from experts on their professional domain-specific languages; to use nonnumeric information without any transformations; to check the consistency of decision maker solutions and eliminate contradictions defined; to explain the solution made $[9,10]$.
Next, let's consider the ontology model that defines the task model, and the algorithm for coordinating expert opinions based on the given ontology and group decision-making methods.

\section{ONTOLOGY MODEL FOR THE MULTIDISCIPLINARY TASK}

The proposed ontology model (Ont) uses concepts of domain (subject) and problem ontologies [11-13].

The problem domain models include knowledge on the ways to solve various types of tasks. At the same time, an effective solution of each task requires adequate representation of data and knowledge, as well as algorithms that implement the solution.

A formal description of the task ontology is proposed in [7] and has the following form:

$$
\begin{aligned}
\text { Ont }{ }^{\text {Problem }}= & \left\langle P N, B T_{-} P, \text { Goal }, C N^{P}, L^{P}, M N, R^{P}\right\rangle \\
& \text { Ont }{ }^{\text {Problem }} R_{\text {use }}{ }^{\text {Ont }} \text { Ont } t^{\text {Method }}, \\
& \text { Ont }{ }^{\text {Problem }} R_{\text {use }}{ }^{\text {Ont }}{ }^{\text {Ont }}{ }^{\text {Subject, }},
\end{aligned}
$$

where Ont ${ }^{\text {Problem }}$ is the ontology of problem area, $P N$ is a multitude of tasks; Goal is a goal of the task, $C N^{P}$ are task concepts, Input ${ }^{P} \cup$ Output ${ }^{P} \subseteq C N^{P}$, Input ${ }^{P}$ are input (source) data and knowledge of the task, Output ${ }^{P}$ are output data and knowledge gained as a result of the task solution, $L^{P} L^{P}$ are task regularities, $M N$ are method names, $R^{P}$ are relationships between tasks and concepts, regularities, methods, $R_{\text {use }}^{\text {Ont }}$ is a use relationship, which in this case means that 
ontology uses the another ontology to identify itself, i.e. ontology of tasks uses concepts of ontologies of methods and disciplines to determine self components.

The ontology of methods is intended to describe methods without taking into account information on the subject and problem areas.

Let's describe methods' ontology. In fact, this ontology describes types of methods that can later be used to create ontologies of problem and application.

$$
\begin{aligned}
& \text { Ont }{ }^{\text {Method }}=\left\langle M N, B T \_M, \text { Input }^{M}, \text { Output }{ }^{M}, L^{M}, A l g,\right. \\
& \text { Soft, } Q, R^{M}>
\end{aligned}
$$

where Ont $t^{\text {Method }}$ is a methods ontology, $M N$ is a method name, $B T \_M$ are the basic methods of ontology, Input ${ }^{M}$ are input (source) data and knowledge of the method, Output ${ }^{M}$ are output data and knowledge gained as a result of the method, $L^{M}$ are regularities defined by the method, Alg is a method algorithm, Soft is a multitude of software that implements the methods, $Q$ is a multitude of indicators describing the method quality, such as speed, accuracy, expert preferences etc., $R^{M}$ is a multitude of relationships, which are define correspondences between the following entities: the method and regularities, the method and concepts, the method and the algorithm of its implementation, the basic method and software of its implementation, respectively.

Let's specify the algorithm description: $A l g=\left(\right.$ Item,$\left.R^{A}\right), \quad$ Item $=\left(\right.$ NItem $\left., B T \_I t e m, B T \_P, B T \_M\right)$ are algorithm stages, NItem is a name of the stage, BT_Item are basic types of algorithm stages including the stage of tasks addressed by basic methods, stages of a cycle type, conditions, aggregation and segregation, $B T_{-} P$ is a basic task type, $R^{A}$ is a multitude of relationships between algorithm stages: sequence, cyclic nature, aggregation, segregation and conditions respectively, these relationships complement a multitude of typical relationships.

It is proposed to use ontology of disciplines to describe multidisciplinarity of tasks. This ontology reflects information related to the discipline considered, its concepts, regularities, tasks:

$$
\text { Ont } t^{\text {Subject }}=\left\langle S N, C N^{S}, P^{S}, M P^{S}, L^{S}, R^{S}\right\rangle,
$$

where $S N$ is the name of the discipline, $P$ are discipline tasks that may have sub-tasks of a basic type, $M P^{S}$ are multidisciplinary tasks highlighted as a particular kind of tasks using ontologies of various disciplines, $C N^{S}$ is a multitude of discipline concepts, $L^{S}$ is a description of regularities postulated by the discipline, $\quad L=\left(C N^{L}, R^{D}, L F, L L\right), \quad L F:\left(C N^{L}, R^{D}\right) \rightarrow L L$, $C N^{L}$ is a multitude of concepts used to describe regularities, $L F$ are some rules for translation of ontology elements to a formal language $L L, C N^{L} \subseteq$ $C N, R^{S}$ is a multitude of relationships between disciplines, concepts, tasks and regularities.

The proposed model provides representation and storage of information about multidisciplinary tasks.

\section{METHOD OF EXPERTS' KNOWLEDGE COORDINATION}

The self-organization algorithm for solving a multidisciplinary task (SAlg from (1)) allows one monitoring the state of the task and generating a solution plan based on the values of indicators (Ind) (potential functions) characterizing the state of the current task and local rules that are the basis of selforganizing behavior.

Let's presents indicators (which together define the aggregated indicator) reflecting the state of the task and independent of its subject (Ind):

- The task status indicator $\left(I^{S P}\right)$ reflects the task solution status, its possible values are: solved, not solved or partially solved;

- The incompleteness of the task elements indicator $\left(I^{U n}\right)$ reflects inaccuracies, uncertainties of the task caused by new ideas from specialists of other disciplines regarding the use of certain data, knowledge (causeeffect relationships), methods of solution, etc .;

- The new task formulation stage indicator $\left(I^{\text {DefP }}\right)$ means either the task at the discussion stage, either that the formulation of the task is completed.

The incompleteness indicator also reflects the aggregated opinion of the team on the status of the elements of the task. The description of the algorithm of its formation is the goal of the work.

Next, we present a local rule (LRule) that forms the indicator to define the position of the knowledge approval method in the process of task solving:

If there is an incompleteness, then algorithms for identified incompleteness reduction are launched.

These algorithms depend on the type of incompleteness:

- incompleteness of the initial data (Input ${ }^{P}$ from (2)), which occurs when a specialist from another discipline, who has not previously participated in the formation of the task, believes that when formulating the task some additional data were not taken into account. In this case, the system creates a new wording of the task and provides the possibility of entering new data into the structure of the task. The new task is under discussion until the coordinator of its decision approves the formulation of the task based on the results of the discussion with all the experts involved in its solution, including the experts who raised the question of the need to use additional data (the occurrence of uncertainty). Then an attempt is made to solve the problem with new data. As a result of the solution, a situation is possible when the existing methods for solving the problem in question are not adequate to the new formulation, then an uncertainty of knowledge $\left(L^{P}\right)$ arises and a transition to the removal of this uncertainty. If the new 
formulation of the problem was approved, then it replaces the previous formulation of the problem;

- incompleteness of knowledge $\left(L^{P}\right)$, which occurs when specialists from other disciplines believe that when formulating a task, some regularities of an analytical or other type are not taken into account. In this case, the system creates a new wording of the task based on the existing one and offers the possibility of introducing new knowledge into the task structure. The new task is under discussion until the resolution of uncertainty;

- incompleteness of methods $(M N)$ occurs when a specialist from another discipline believes that other possible methods of solving the problem can be used to solve the problem. In this case, the system prompts the specialist to describe a new method for solving the problem and indicate either an existing tool (Soft from (3)) implementing the method or specify a new tool. The formulation of the new method is under discussion until the resolution of uncertainty.

The following principles of interaction between experts in resolving the listed types of incompleteness are formulated:

- experts can suggest changes in the data, regularities, methods and means (possible actions: edit, add, delete) of any task;

- experts should argue their proposals (argumentation based on experience, experiment, theoretical justification). The arguments must be stored for further processing and use;

- experts should be able to ask clarifying questions. The dialogue should be accessible for the team of experts;

- the team of experts should be notified of all changes;

- approval of opinions should has an iterative character, i.e. discussion takes place until the moment when a certain criterion is fulfilled (for example, the majority criterion);

- the conclusion of the discussion is a survey of experts on agreement with the changes.

Let's formalize the concepts of the method based on the principles above:

\footnotetext{
$<$ Incompleteness approval method $>=$ (<Incompleteness $>$ <Expert team> <Expert opinion $><$ Approval methods $>$ )

$<$ Expert team $>=<$ Expert $>\{<$ Expert $>\}$

$<$ Expert $>\quad=\quad$ Expert name $><$ Expert knowledge area $>\{<$ Expert knowledge area $>$ <Expert actions $>$
}

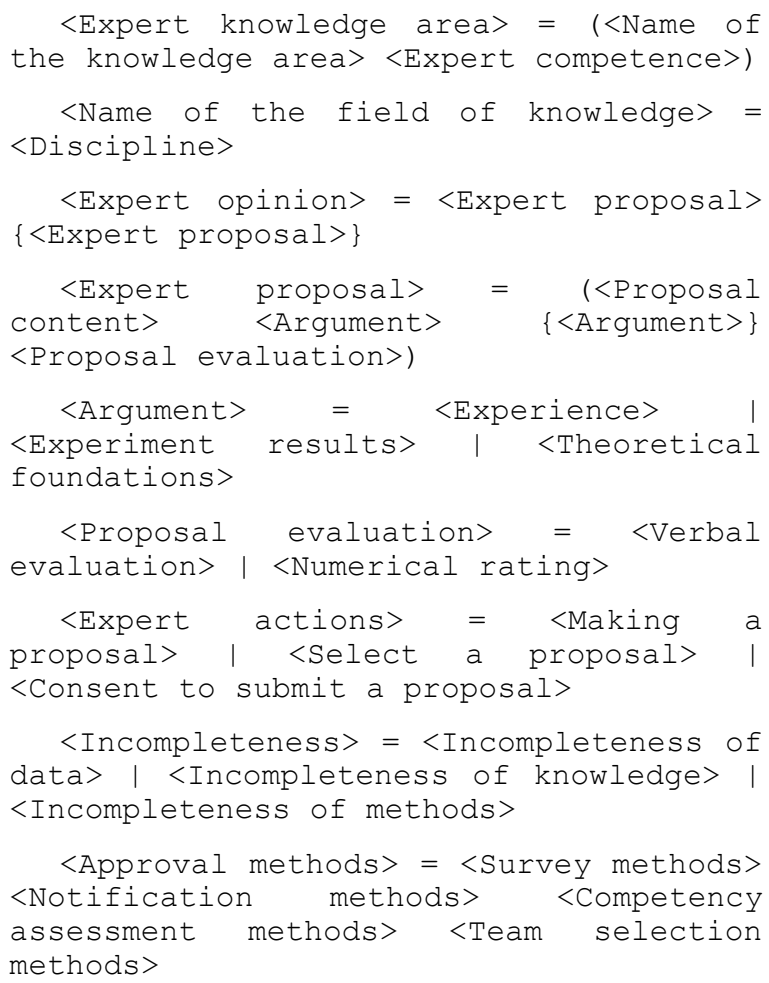

The proposed formalization of concepts provides storing and exchange of data between DSS components (see 1).

The algorithm (Fig. 2) allows one to solve the listed types of incompleteness on the basis of the proposed principles.

\section{EXAMPLE OF DESCRIBING THE TASK MODEL}

Let's consider the examples of the elements of the interdisciplinary task model for diagnosing TD technical states:

- disciplines: $S N=\{$ material resistance, fracture mechanics, corrosion resistance, heat resistance, chemical resistance, wear resistance, physical and chemical fracture mechanics, ... $\}$,

- $\quad$ input information for the task Input ${ }^{P}=\{\mathrm{TD}$ properties / parameters and their values $\}$,

- $\quad$ output information of the task Output $^{P}=$ \{properties / parameters of the technical state of TD and their values ,

- $\quad$ methods of the task: $M N=\{$ case-based expert system, rule-based expert system, ... $\}$,

- $\quad$ regularities of the task: $L^{P}=\{$ knowledge base of degradation processes, ... $\}$,

- a model of a case-based expert system for identification of technical states is based on the basic model of this method: $M N^{C B R}=<M N=C B R, B T \_M=C B R$,

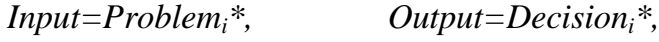
$L=\left\{\right.$ Case $\left._{i}\right\}, \quad$ Alg=k-means, $\ldots>$, where Case $_{i}=\left\langle\right.$ Problem $_{i}$, Decision $\left._{i}\right\rangle$, the problem is described by a set of tuples Problem $_{i}=<p_{i j}$, 
$v_{i j}, r_{i j}>$, where $p_{i j}$ is the name of the $j$-parameter, $v_{i j}$ is the value of the $j$-parameter, $r_{i j}$ is a constraint for the value of the $j$-parameter, solution is a set of parameters similar in structure describing a solution; Problem $_{i}{ }^{*}$ is a description of the initial situation / problem; Decision $_{i}{ }^{*}$ is a solution proposed as a result of the execution of the algorithm of the method,

- the model of the rule-based expert system for identification of the technical state is based on the basic model of this method: $M N^{R B R}=<M N=R B R, B T \_M=R B R$, Input $=\left\{<p_{j}\right.$, $\left.v_{j},>\right\}, \quad$ Output $=\left\{\left\langle p_{j^{*},} \quad v_{j^{*}}\right\rangle\right\}, \quad L=\left\{\mathrm{IF}<p_{j 1}\right.$, $v_{j 1},>\circ \ldots \circ<p_{j N}, v_{j N},>$ THEN $\left\langle p_{j^{*}}, v_{j^{*}},>\right\}$, Alg $=$ "rule-based reasoning", ... , $\left\langle p_{j}, v_{j},\right\rangle$ is an initial fact, $\left\langle p_{j^{*}}, v_{j^{*}}\right\rangle$ is a fact resulting from the execution of the method,

- the task hierarchy contains sub-tasks for diagnosing a technical state: the task of identification of the current state, the task of the genesis of the technical state. The genesis task also has sub-tasks for identification of the technical state for the classes of dynamics of degradation processes: the initial state $\rightarrow$ the admissible state $\rightarrow$ the limiting state $\rightarrow$ the state of destruction, etc.

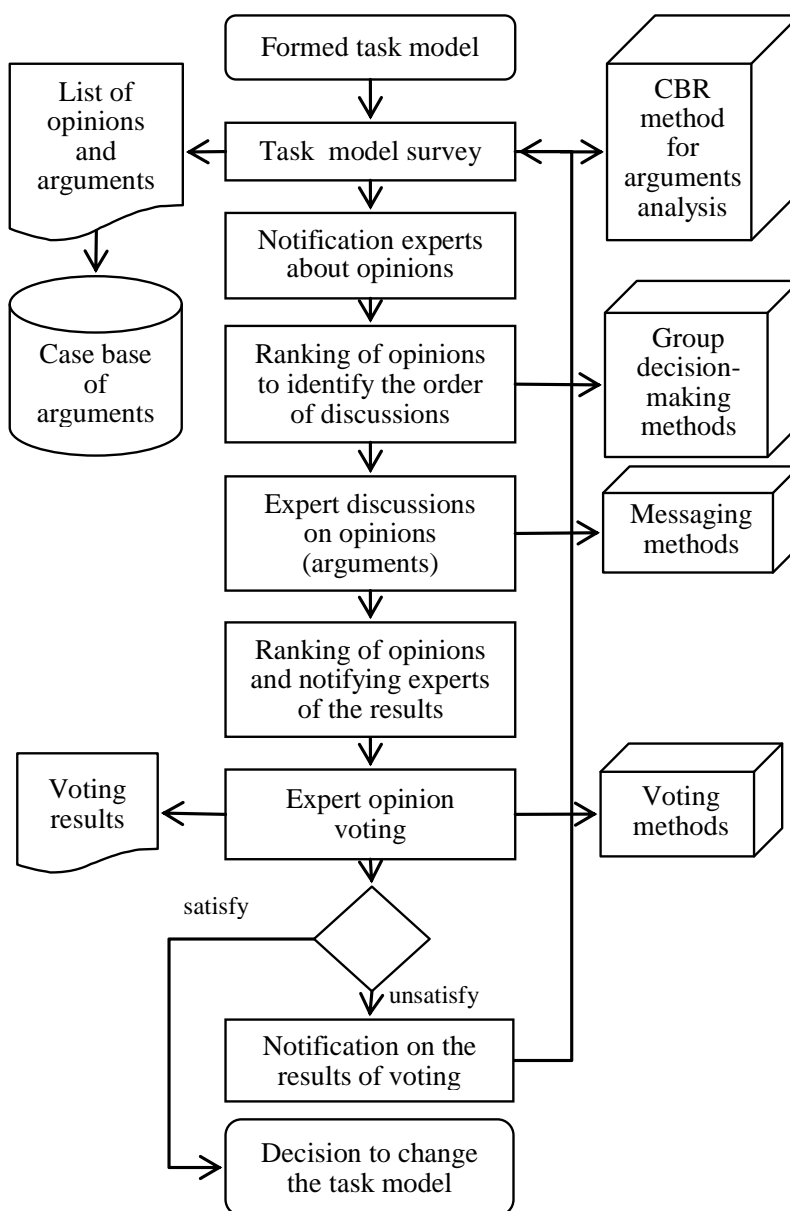

Fig. 2. The algorithm for approval of experts' opinions in resolving incompleteness.

\section{EXAMPLE OF THE APPLICATION OF THE EXPERTS' KNOWLEDGE APPROVAL METHOD}

Let's describe and example of the application of the experts' knowledge approval method for definition of a logical rule for a degradation process (DP) mechanism in the case of diagnosing the TD technical state task. Types of DP and factors (facts) characterizing a certain mechanical system and the conditions of its operation are known. It is necessary to coordinate the opinions of experts (perhaps representatives of various disciplines) about particular facts, which are determine the given DP and will be included in the rule.

The approval of knowledge is carried out consistently in accordance with the structure of knowledge in the rules (tabl. 1):

- Level 1 - approval of the facts that should be taken into account when building the left part of the rules ("condition"), only the "AND" operator allowed between facts;

- Level 2 - formation of an approved list of parameters that affect each fact;

- Level 3 - approval of scales (values) for each parameter.

In order to reduce the expert effort it is proposed to automate the approval process, leaving the experts the right to agree with the results or reject them.

Let's consider the approval process in detail. At the first level each expert speaks with arguments about the facts that need to be taken into account. The expert opinion is represented in the form of a triple $<$ Proposal content $=$ Fact, Fact evaluation, Competence evaluation >, where evaluation of the fact is formed on the basis of verbal expressions: the fact is not supported (ns), there is no opinion for this fact (no), the fact is supported (s); the evaluation of competence also has verbal values: there is no competence (nc), the competence is limited (lc), the competence is high (hc). Various methods can be used to assess the competence of each expert, for example, ARAMIS [14]. Then, a multiset is built on the basis on polls of all participants in the discussion, and an aggregated score is determined using ARAMIS, in particular $F_{1} \rightarrow F_{3} \rightarrow F_{2}$ (tabl. 2). This ordering is presented to the experts for the approval / rejection of the list of facts that need to be taken into account. Voting results are processed according to the principle of simple majority of votes. The parameters of the facts and their values are approved by the same way at the second and third levels.

\section{CONCLUSION}

The presented results make it possible to organize (nd automate) the approval of opinions (knowledge) of experts from various subject areas when formalizing and solving industrial safety expertise tasks. The proposed approach is tested in solving tasks of identification and prediction of degradation processes occurring in the unique mechanical systems of oil critical infrastructures. 
TABLE 1. AN EXAMPLE OF THE STAGES OF THE EXPERT KNOWLEDGE APPROVAL

\begin{tabular}{|c|c|c|c|}
\hline \multicolumn{3}{|c|}{ Factors } & \multirow[t]{2}{*}{ Type of DP } \\
\hline Medium $\left(F_{1}\right)$ & Material $\left(F_{2}\right)$ & Loads $\left(F_{3}\right)$ & \\
\hline \multicolumn{3}{|c|}{ Approval Level 1} & \multirow{6}{*}{$\begin{array}{l}\text { Corrosion } \\
\text { cracking }\end{array}$} \\
\hline $\begin{array}{l}\text { Heat exchange } \\
\text { medium }\end{array}$ & Residual stresses & Mechanical loads & \\
\hline & Approval Level 2 & & \\
\hline Hydrogen indicator & Type, value, location, orientation, reason & Type, value & \\
\hline \multicolumn{3}{|c|}{ Approval Level 3} & \\
\hline $\begin{array}{c}\text { Hydrogen indicator }< \\
10\end{array}$ & $\begin{array}{l}\text { Type - stretching; value - maximum; location - on the side } \\
\text { OR the concave surface of the pipe; orientation - } \\
\text { longitudinal; reason - cold bending deformation }\end{array}$ & $\begin{array}{l}\text { Type - constant, value > } \\
\text { yield point of the part } \\
\text { material }\end{array}$ & \\
\hline
\end{tabular}

TABLE 2. AN EXAMPLE OF A MULTISET BUILDING AND OBTAINING AN AGGREGATED SCORE

\begin{tabular}{|c|c|c|c|c|}
\hline & \multicolumn{3}{|c|}{ Factors } & \multirow{4}{*}{ Expert competence } \\
\hline & Medium $\left(F_{1}\right)$ & Material $\left(F_{2}\right)$ & Loads $\left(F_{3}\right)$ & \\
\hline & \multicolumn{3}{|c|}{ ApprovalLevel 1} & \\
\hline Facts & Heat exchange medium & $\begin{array}{c}\text { Residual } \\
\text { stresses }\end{array}$ & $\begin{array}{c}\text { Mechanical } \\
\text { loads }\end{array}$ & \\
\hline Expert 1 & $\mathrm{~S}$ & $\mathrm{~s}$ & $\mathrm{~s}$ & hc \\
\hline Expert 2 & $\mathrm{~S}$ & no & $\mathrm{s}$ & lc \\
\hline Expert 3 & $\mathrm{~S}$ & no & $\mathrm{ns}$ & lc \\
\hline Multiset & $\begin{array}{l}\left\{3^{\circ} \mathrm{s}, 0 \circ \mathrm{no}, 0 \circ \mathrm{ns}\right. \\
1 \circ \mathrm{hc}, 2 \circ \mathrm{lc}, 0 \circ \mathrm{nc}\}\end{array}$ & $\begin{array}{l}\{1 \circ \mathrm{s}, 2 \circ \mathrm{no}, 0 \circ \mathrm{ns} \\
1 \circ \mathrm{hc}, 2 \circ \mathrm{lc}, 0 \circ \mathrm{nc}\}\end{array}$ & $\begin{array}{l}\left\{2^{\circ} \mathrm{s}, 0 \circ \mathrm{no}, 1 \circ \mathrm{ns} ;\right. \\
1 \circ \mathrm{hc}, 2 \circ \mathrm{lc}, 0 \circ \mathrm{nc}\}\end{array}$ & \\
\hline $\begin{array}{l}\text { Aggregated score } \\
\text { (ARAMIS) }\end{array}$ & 0.25 & 0.4 & 0.375 & \\
\hline
\end{tabular}

\section{ACKNOWLEDGMENT}

The reported study was partially supported by RFBR grants №18-07-01164, 18-08-00560.

\section{REFERENCES}

[1] A.F. Berman, O.A. Nikolaichuk, A.Y. Yurin and K.A. Kuznetsov, "Support of Decision-Making Based on a Production Approach in the Performance of an Industrial Safety Review,“ in Chemical and Petroleum Engineering, vol.50, no.1-2, 2015, pp.730-738.

[2] I. Z. Aronov , O. V. Maksimova , A. V. Zazhigalkin "Investigation of time to reach consensus on the work of technical committees on standardization based on regular Markov chains," in Computer Research and Modeling, vol. 7 , no. 4, 2015, pp. 941-950.

[3] P. Levy, "Frequently asked questions about collective intelligence".

URL: http://web.archive.org/web/20030215235346/http://ww w.collectiveintelligence.info/cifaq.htm\#top (accessed on 15.01.2018).

[4] I. A. Kirikov, A. V. Kolesnikov, S. V. Listopad, and S. B. Rumovskaya, "Virtual heterogeneous collectives for supporting decision-making," in Systems and Means of Informatics, v. 25, no 3, 2015, pp. 126-149.

[5] D. Gubanov, N. Korgin, D. Novikov, and A. Raikov, EExpertise: Modern Collective Intelligence. / Series:Studies in Computational Intelligence. vol. 558.-Springer International Publishing, 2014, pp. 112

[6] Yu.V. Sidelnikov, System analysis of expert forecasting. Moscow.: MAI, 2007, 453 p.

[7] A.F. Berman, O.A. Nikolaychuk, and A.I. Pavlov "The Ontology Model for Automating the Solution of
Multidisciplinary Research Tasks," in Proc. the V Intern. Workshop Critical Infrastructures: Contingency Management, Intelligent, Agent-Based, Cloud Computing and Cyber Security (IWCI 2018), vol. 158, 2018, pp. 1-6.

[8] A.F. Berman, O.A. Nikolaichuk, and A.I. Pavlov "Selforganizing decision-making algorithm to ensure the required technical condition of complex hazardous objects," in 7th International Conf. "System Analysis and Information Technologies", (June 13-18, 2017, Svetlogorsk, Russia): Conference proceedings, 2017, pp. 377-384.

[9] A.B. Petrovsky, "Group verbal decision analysis," in: Adam, F., Humphreys, P. (eds.) Encyclopedia of Decision Making and Decision Support Technologies, IGI Global, Hershey, 2008, pp. 418-425.

[10] A. B. Petrovsky, "Group Multiple Criteria Decision Making: Multiset Approach," in Recent Developments and New Directions. Soft Computing, Springer, Cham, 2014, pp. 1933.

[11] T.A. Gavrilova, and I.A. Leshcheva "Ontology design and individual cognitive peculiarities: A pilot study," Expert Systems with Applications. 42(8):3883-3892. doi:10.1016/j.eswa.2015.01.008.

[12] B. Chandrasekaran, J.R. Josephson, and V.R. Benjamins "Ontology of Tasks and Methods," IEEE Intelligent Systems, 14(1), 1998, pp. 20-26.

[13] R. Studer, R. Benjamins, and D. Fensel, "Knowledge Engineering: Principles and Methods," in Data and Knowledge Engineering, 25(1-2), 1998, pp. 161-197.

[14] G.S. Maltugueva, and A.Y. Yurin, "Combination of the group and multi-criteria decision-making methods," in Business management in Decision-Making: Processes, Behavioral Influences and Role, Nova Science Publishers, Inc., 2015, pp. $1-13$. 